\title{
Microvasculature of Anterior Cruciate Ligament by Immunohistochemistry using VE-Cadherin
}

\author{
Jahira Banu T1, Yogesh Ashok Sontakke \\ 1Junior Resident, Department of Anatomy, JIPMER, Pondicherry, India, ${ }^{2}$ Associate professor, Department of Anatomy, JIPMER, Pondicherry, India.
}

\section{Abstract}

Introduction: The anterior cruciate ligament (ACL) is one of the knee stabilizer and acts to prevent excessive anterior mobility as well as rotational movement. The ACL is extending from the lateral femoral condyle to the anterior intercondylar area of tibia. During excessive movement or abnormal mobility affecting the knee joint, the ACL is under tension and prone for injury. The injured ACL was managed by surgery as it was an option for treatment owing to its poor vascularity. The distribution of the blood vessels within the ACL was not clear and only few studies reported in the past. The present study focused on distribution of blood vessels in the ACL. Subjects and Methods: The ACL microvasculature was assessed using 48 cadaveric ACL tissues using immunohistochemistry. The antibody against the transmembrane protein VE-Cadherin was targeted to study the blood vessels. Results: It was observed that the middle part of the ACL was less vascular compared with the peripheral parts of ACL. Conclusion: The knowledge of the ACL vasculature may help in planning surgeries of ACL to reduce postoperative complications.

Keywords: Anterior Cruciate Ligament, Immunohistochemistry.

Corresponding Author: Dr. Yogesh Ashok Sontakke, Associate professor, Department of Anatomy, JIPMER, Pondicherry, India. Email: dryogeshas@gmail.com

\section{Introduction}

The Anterior cruciate ligament (ACL) is acting as one of the knee stabilizing structure and prevents excessive anterior mobility and abnormal rotation of the knee joint. The ACL is an intra-articular structure extending from the lateral femoral condyle to the anterior intercondylar region of tibia. It is the most common ligament to be affected by knee injury. ${ }^{[1]}$

The ACL undergoes injury during a direct hit to the knee as well as in indirect contact such as in sports or in faulty landing. Indirect injury was a more common type of insult to the ACL. ${ }^{[2]}$ The injured ACL was repaired by reconstructive procedure using tendon grafts. The reason for the reconstruction of ACL instead of repairing the ACL ends with suture was due to poor healing capacity of ACL. ${ }^{[3]}$ Although the ACL injury was repaired by replacing tendon, in many of the case it produces a failure in surgery. The replaced tendon was not structurally similar with the ACL ligament, often the reconstructed tendon cannot reproduce the functions of ACL.

Therefore, to reproduce the functional aspects of the ACL, the presence of native ACL is needed. The vasculature knowledge of ACL is important to understand the healing ability of ACL. The ACL received its blood supply from the middle geniculate artery, a branch of popliteal artery. Its subdivides when it reaches the synovial ligament of the ACL. The subdivided branches further enter the ligaments and supplies the ligament substance.

Toy et al., found out that the ACL has more vascularity in the proximal and distal parts compared with the middle part of the ACL. They described that ACL has less intraligamentous blood vessels than the surrounding connective tissue. ${ }^{[4]}$ Peterson et al come out with the results of avascular region in the distal part of the ACL and more vascular region in the remaining parts of ACL. ${ }^{[7]}$

From the previous studies it was observed that, some part of the ACL is avascular. Therefore, we aimed at to study the microvasculature of ACL using immunohistochemistry. The VE-Cadherin antibody, a transmembrane protein located in the adjacent endothelial cells was targeted to localize the blood vessels immunohistochemically. It is responsible for maintaining the continuity of endothelial cells. The adhesive property of this complex act as a selective barrier. The barrier maintained by the VE-cadherin can be disrupted by the bacterial invasions and can precipitate sepsis, oedema and haemorrhages. ${ }^{[11,12]}$ The regions of ACL with more vascularity can be repaired with the biological augmentation procedure and the avascular part can be corrected with the tendon replacement surgery.

\section{Subjects and Methods}

The required study sample was collected from the Anatomy department of about 48 limbs. All embalmed cadavers fit to be dissected were included in the study. Cadavers with any evidence of damage or loss of tissues in the region of knee joint were excluded. The knee joint was approached anteriorly for the dissection of ACL. A curvilinear incision 
was made transversely over the lower end of quadriceps muscles and the patella was reflected downwards. The knee joint cavity was made exposed and the infrapatellar pad of fat was removed until the ACL was made visible. The ACL was dissected out from the most proximal region to the most distal attachment. The ACL was divided equally into three parts (segments) according to the length of the ACL. The ACL parts were subjected to histological processing and paraffin embedded blocks were prepared.

For the immunohistochemistry procedure, the tissue was sectioned at $5 \mu \mathrm{m}$ thickness using thermo-scientific microtome. ACL segments were rehydrated and the sections were processed for antigen retrieval using retrieval buffer. The sections were then stained with primary antibody, VECadherin antibody (host; mouse, Novus biologicals, 1:1000 dilution) and secondary antibody coated with Cy 5 (goat antimouse IgG tagged with Cy5, Novus biologicals, 1:1000 dilution). The slides were then mounted with 1S PBS and the edges sealed with nail polish. Then the slides were analyzed under microscope or stored temporarily under refrigerator $\left(4-8^{\circ} \mathrm{C}\right)$ till visualization. (10) The confocal microscope (Carl Zeiss LSM 700) with the zen-software 2010 was used for creating and analysing the microscopic images. The blood vessels fluoresced in reddish colour and the image was analysed with ZEN software. The blood vessel luminal diameter was calculated using ImageJ software. The statistical analysis involving the comparison of luminal diameters within the ligaments and between each parts were analysed using SPSS software version 20.

[Figures 1] shows immunofluorescence image of blood vessel against VE-Cadherin and [Figure 2] shows the measurement of luminal diameter of blood vessel using ImageJ software.

\section{Results}

The parameters evaluated was number of blood vessels and the luminal area of blood vessels in the ACL. The luminal areas of blood vessels from the three parts of ACL obtained were tabulated. The average values in the proximal part, middle part and distal part were $8369.10 \mu \mathrm{m} 2,5590.97 \mu \mathrm{m} 2$, $6724.05 \mu \mathrm{m} 2$ respectively. There was a statistically significant difference in the luminal area of blood vessels between the three parts of ACL (p-value < 0.01) as determined by using one-way ANOVA analysis tests [Table 1]. It was observed that there was a significant difference between the luminal area of ACL in between the proximal and middle parts with mean difference of $2778.14 \mu \mathrm{m} 2$; between the proximal and distal parts with mean difference of $1645.06 \mu \mathrm{m} 2$ and also between the middle and distal parts with mean difference of _ $1133.09 \mu \mathrm{m} 2$. [Table 2].

The average number of blood vessels in the proximal, middle and distal parts of ACL were 17, 12 and 16 respectively. [Table 3] There was a statistically significant difference in the number of blood vessels between the three parts of ACL as determined by one-way ANOVA test. It was observed that there is a significant difference between the number of blood vessels in the proximal and middle parts of ACL and the mean difference is 6 . There is also a significant difference between proximal and distal parts and the mean difference is 3. There is a significant difference between the middle and distal parts of ACL, and the mean difference is _ 3. [Table 4].

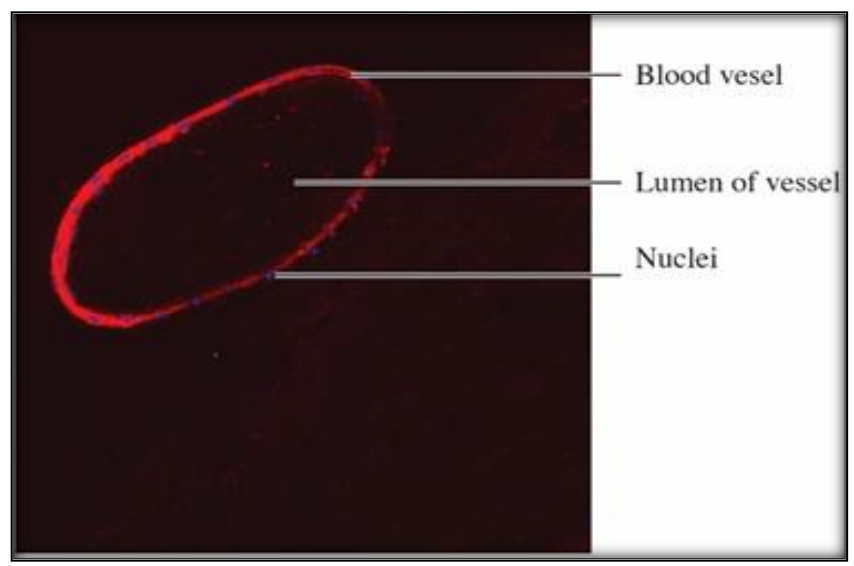

Figure 1: Blood vessel (X400, Stain: Nuclei - DAPI, Endothelium: VE-Cadherin primary antibody, Cy-5 tagged secondary antibody)

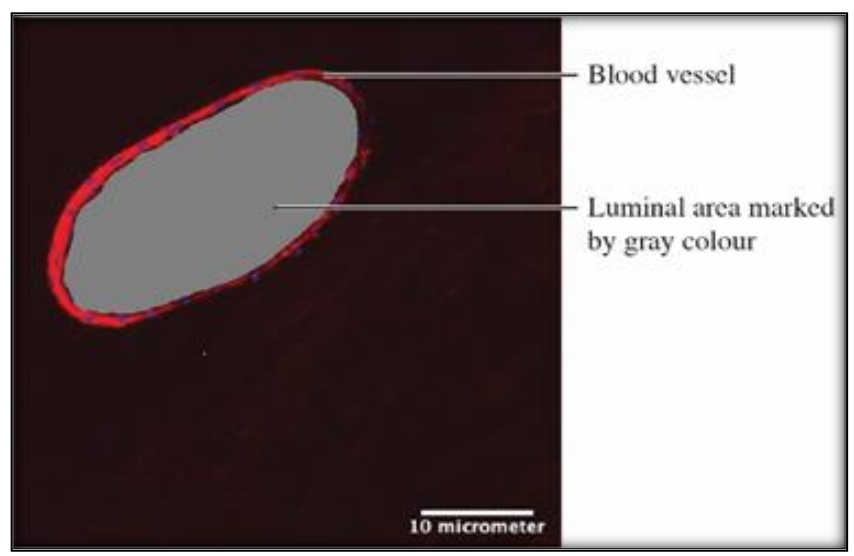

Figure 2: Measurement of luminal area of blood vessel in IHC image using ImageJ software version $1.52 q$ (X400, Stain: Nuclei - DAPI, Endothelium: VE-Cadherin primary antibody, Cy-5 tagged secondary antibody)

Table 1: Measurement of blood vessel luminal area in the three parts of ACL by IHC

\begin{tabular}{|l|l|l|}
\hline Parts of ACL & BV LA IHC $\left(\boldsymbol{\mu m}^{\mathbf{2}}\right)$ & $*$ p-value \\
\hline Proximal & $8369.10 \pm 1841.81$ & $25(21-30)$ \\
\hline Middle & $5590.97 \pm 2321.45$ & $<0.01$ \\
\hline Distal & $6724.05 \pm 1935.97$ & $235(95.9 \%)$ \\
\hline \\
*One -way ANOVA test, BV LA IHC - blood vessels luminal area \\
immunohistochemistry.
\end{tabular}

Table 2: Blood vessel luminal area difference between the three parts of ACL by IHC

\begin{tabular}{l|l|l|l|l|}
\hline $\begin{array}{l}\text { Dependent } \\
\text { variable }\end{array}$ & \multicolumn{2}{|l|}{ Parts of ACL } & $\begin{array}{l}\text { Mean } \\
\text { difference }\end{array}$ & $\begin{array}{l}\text { *p- } \\
\text { value }\end{array}$ \\
\hline \multirow{2}{*}{$\begin{array}{l}\text { BV LA } \\
\text { IHC }\end{array}$} & Proximal & Middle & 2778.14 & $<0.001$ \\
\cline { 2 - 5 } & & Distal & 1645.06 & $<0.001$ \\
\cline { 2 - 5 } & Middle & Distal & -1133.09 & $<0.001$ \\
\hline \multirow{2}{*}{$\begin{array}{l}\text { *Bonferroni post-hoc test, BV LA IHC - blood vessel luminal area } \\
\text { immunohistochemistry }\end{array}$}
\end{tabular}

Table 3: Measurement of number of blood vessels in three parts of ACL by IHC

\begin{tabular}{|l|l|l|}
\hline Parts of ACL & BV NO IHC $($ Mean \pm SD) & $*$ p-value \\
\hline Proximal & $17.96 \pm 3.63$ & \\
\hline Middle & $11.83 \pm 2.95$ & $<0.001$ \\
\hline Distal & $14.56 \pm 2.29$ & \\
\hline
\end{tabular}


Table 4: Difference in the number of blood vessels between the ACL parts by IHC

\begin{tabular}{|l|l|l|l|l|}
\hline Parameter & \multicolumn{2}{|l|}{ Parts of ACL } & $\begin{array}{l}\text { Mean difference in } \\
\text { number of blood } \\
\text { vessels }\end{array}$ & *p-value \\
\hline & Proximal & Middle & 6.19 & $<0.001$ \\
\hline BV NO & & Distal & 3.48 & $<0.001$ \\
\cline { 2 - 5 } IHC & Middle & Distal & -2.79 & $<0.001$ \\
\hline
\end{tabular}

*Bonferroni post-hoc test

\section{Discussion}

The ACL consists of many collagen bundles that runs in a random direction and gives a complex appearance when viewed microscopically. The complex arrangement of collagen bundles in the ACL helps in providing different actions pertaining to the ACL. Marshall et al studied the ACL vascularity using Indian ink injection method and found out that the entire ACL was vascular and there is avascular zone near the attachment site. Toy et al studied the ACL vasculature using $\mathrm{CT}$ scan and dissections and found out that the middle zone of ACL was less vascular. The proximal and distal zone was vascularized more. The blood vessels in the proximal part showed more tortuosity than the distal part of the ACL. ${ }^{[4]}$

Scapinelli assessed the ACL vasculature using the radiography, indian ink injection method and histology. On comparison with the three methods, they discovered that the extremes of ACL were vascularized more and less vascular region in the junction of middle and distal zone and also in the attachment site. ${ }^{[5,6]}$ Peterson et al compared the two methods of ACL using two methods such as injection method by Indian ink and immunohistochemistry. They identified that the ACL has an avascular portion in the distal region. ${ }^{[7]}$ Hetsroni et al assessed the ACL vasculature from the patients underwent knee arthroscopy. They pointed out that the ACL vascularity was varying among the different age groups. The ACL of age group less than 30 years has more vascularity in the entire ligament. The age group of 40-60 years has avascular zone in the middle part of the ligament. ${ }^{[9]}$

By using VE- cadherin the specific antibody against the blood vessels, we studied the ACL vasculature. Our observation from the vasculature search throughout the ACL ligament showed a wide variation in the distribution of vessels. The proximal part of the ligament is more vascular compared with the remaining parts of ACL. The middle part of the ACL has less vascularity relative to the remaining parts of ACL. The intra-ligamentous distribution of blood vessels was less compared with the blood vessels in the surrounding connective tissue. Therefore, comparing with the previous studies on ACL vasculature present study found out that the ACL has no avascular part. Injury affecting ACL vascular parts can be treated with the native ACL without reconstruction with tendon transfer. The present research can be extended by using samples from the postoperative specimens and arthroscopy cases.

\section{Conclusion}

The present study documented that the ACL vasculature using VE-Cadherin antibody. The ACL has more vascularity in the peripheral regions compared with the middle part of the ACL. The middle part is less vascular than the other two parts of ACL. The blood vessels were less in the intraligamentous region compared with the periphery of the ligament. The reason for the improper healing of ACL may be due to the less vascularity in the intra-ligament portion of the ACL. The treatment of injured ACL involving the vascular region can be repaired with biological augmentation procedures without replacing ligament.

\section{References}

1. Gordon MD, Steiner ME. Anterior cruciate ligament injuries. In: Garrick JG, ed. Orthopaedic knowledge update sports medicine III. Rosemont: American academy of orthopeadic surgeons. 2004;p.169.

2. Griffin LY, Agel J, Albohm MJ, Arendt EA, Dick RW, Garrett WE, Garrick JG, Hewett TE, Huston L, Ireland ML, Johnson RJ. Noncontact anterior cruciate ligament injuries: risk factors and prevention strategies. JAAOS-Journal of the American Academy of Orthopaedic Surgeons. 2000;8:141-50.

3. Duthon VB, Barea C, Abrassart S, Fasel JH, Fritschy D, Ménétrey J. Anatomy of the anterior cruciate ligament. Knee surgery, sports traumatology, Arthroscopy. 2006;14:204-3.

4. Toy BJ, Yeasting RA, Morse DE, McCann P. Arterial supply to the human anterior cruciate ligament. J Athl Train. 1995;30:149-153.

5. Scapinelli R. Studies on the vasculature of the human knee joint. Cells Tissues Organs. 1968;70:305-31.

6. Scapinelli R. Vascular anatomy of the human cruciate ligaments and surrounding structures. Clin Anat. 1997;10:151-62.

7. Petersen W, Hansen U. Blood and lymph supply of the anterior cruciate ligament: cadaver study by immunohistochemical and histochemical methods. Int. J. Orthop. Sci. 1997;2:313-8.

8. Marshall JL, Arnoczky SP, Rubin RM, Wickiewicz TL. Microvasculature of the cruciate ligaments. Phys Sports med. 1979;7:87-91.

9. Hetsroni I, Manor A, Finsterbush A, Lowe J, Mann G, Palmanovich E. Reduced anterior cruciate ligament vascularization is associated with chondral knee lesions. J Orthop. 2016;39(4):737-43

10. Sontakke YA. Principles of histological techniques, immunohistochemistry and microscopy. 1st ed. India: Paras medical books pvt.ltd; 2017.

11. Sidibé A, Imhof BA. VE-cadherin phosphorylation decides: vascular permeability or diapedesis. Nature immunology. 2014;15(3):215.

12. Iurlaro M, Demontis F, Corada M, Zanetta L, Drake C, Gariboldi M, Peiro S, Cano A, Navarro P, Cattelino A, Tognin S. VE-cadherin expression and clustering maintain low levels of survivin in endothelial cells. The American journal of pathology. 2004;165(1):181-9. 


\section{Banu d Santakke; Micravas culature of Anteriar cruciate ligament ley immunahistachemistry}

Copyright: (C) the author(s), 2020. It is an open-access article distributed under the terms of the Creative Commons Attribution License (CC BY 4.0), which permits authors to retain ownership of the copyright for their content, and allow anyone to download, reuse, reprint, modify, distribute and/or copy the content as long as the original authors and source are cited.

How to cite this article: Banu TJ, Sontakke YA. Microvasculature of Anterior cruciate ligament by immunohistochemistry using VE-Cadherin. Acad. Anat. Int. 2020;6(1):58-61.

DOI: dx.doi.org/10.21276/aanat.2020.6.1.13 